\title{
Protection of Medical Staff during Tracheotomy: Lessons Learned from the COVID-19 Pandemic
}

\author{
Dong-Hyun Lee Subin Kim Ji-Sun Kim Byung Guk Kim \\ Ki-Hong Chang Jun-Ook Park
}

Department of Otolaryngology-Head and Neck Surgery, Eunpyeong St. Mary's Hospital, College of Medicine, The Catholic University of Korea, Seoul, Republic of Korea

\author{
Keywords \\ COVID-19 · Novel coronavirus · Airway management · Tracheotomy $\cdot$ Medical staff \\ protection
}

\begin{abstract}
Background: During the ongoing pandemic of COVID-19, tracheotomy under emergency situation is considered a high-risk procedure that causes probable expose to aerosolized secretion. Summary: We reviewed our case and previous reports, and summarized a detailed protocol that is needed to protect medical staffs who perform tracheotomy under the COVID-19 pandemic, considering the patient's condition, experience of medical staff members, and available facilities and equipment. Key Messages: For efficient protection of medical staff who perform tracheotomy under the COVID-19 pandemic period, we suggest that the following needs to be considered: assessment of patient's condition (COVID-19 infection and the airway problem), route (safest route to the operating room), experienced surgical team, negative-pressure isolation facility and appliance (personal protective equipment) availability, and safe and appropriate post-tracheotomy care.

(C) 2020 The Author(s)

Published by S. Karger AG, Basel
\end{abstract}

\section{Introduction}

The novel coronavirus (COVID-19) belongs to the family Coronaviridae, comprising single-stranded RNA viruses that cause respiratory infections in humans. The outbreak of COVID-19, which started in Wuhan, China, in December 2019, has spread to Southeast Asia, Europe, the United States, and across the world; the World Health Organization (WHO) has 
Table 1. Full search strategy

\begin{tabular}{ll}
\hline$\# 1$ & Coronavirus [Title/Abstract] \\
$\# 2$ & Viral infection [Title/Abstract] \\
$\# 3$ & COVID-19 [Title/Abstract] \\
$\# 4$ & SARS [Title/Abstract] \\
$\# 5$ & MERS [Title/Abstract] \\
$\# 6$ & Search \#1 OR \#2 OR \#3 OR \#4 OR \#5 \\
$\# 7$ & Airway management [Title/Abstract] \\
$\# 8$ & Tracheotomy [Title/Abstract] \\
$\# 9$ & Medical staff protection [Title/Abstract] \\
$\# 10$ & Search \#7 OR \#8 OR \#9 \\
$\# 11$ & Search \#6 AND \#10 \\
\hline
\end{tabular}

declared a global pandemic. Since January 19, 2020, when a Chinese woman infected with COVID-19 entered Korea from Wuhan, a total of 10,564 confirmed COVID-19 cases have been reported in Korea as of April 14, 2020. Some cases are asymptomatic, but many patients present with high fever, respiratory symptoms, and general fatigue. COVID-19 infections in high-risk groups, such as immunocompromised older adults with underlying conditions, can trigger acute respiratory distress syndrome, requiring ventilation therapy in the intensive care unit, and even death [1]. Head and neck surgeons may have to perform emergent or urgent tracheotomy before the results of the COVID-19 PCR test are available, such as in patients with acute epiglottitis, laryngeal cancer, trauma, or tracheal tumor. In this situation, it is essential to protect medical staff from nosocomial infection. As tracheotomy is a surgical opening in the trachea that exposes the airway, the surgeon faces the airway lumen during the procedure and is thus directly exposed to the droplets and aerosol particles generated when the cough reflex is triggered by tracheal stimulation during the procedure or due to the pressure applied by the mechanical ventilator. To protect medical staff from infection, appropriate training and facilities and protective equipment are required. Based on a review of our case report and previously published reports, in the following we provide a detailed protocol for protecting medical staff performing tracheotomy during the COVID-19 pandemic.

\section{Methods}

We searched the literature using PubMed and KoreaMed using the search terms viral infection, coronavirus, COVID, SARS, or MERS, and then airway management, tracheotomy, or medical staff protection. The full search strategy is shown in Table 1 . Articles that could not be obtained in the full text, did not mention a relationship between viral infection and tracheotomy directly, were not written in English, and were duplicated papers by the same author were excluded using Endnote X9 software. There were no restrictions with respect to sex, age, and nationality. Studies that met the inclusion criteria were checked independently by two reviewers. If the title and abstract met the inclusion criteria, the full text was checked by the same reviewers.

\section{Case}

An 87-year-old man with tracheal squamous cell carcinoma visited our hospital for dyspnea. He had a known 2.0-cm tracheal squamous cell carcinoma in the posterior wall of the subglottic trachea, detected previously by bronchoscopy. A COVID-19 PCR test was performed but, even in the absence of its results, we decided to perform elective tracheotomy under local anesthesia, given the possible deterioration of the patient's dyspnea symptoms. Tracheotomy was performed by an experienced head and neck surgeon, a surgical assistant, a scrub nurse, and a circulating nurse, all of whom wore level D personal protective equipment (PPE) and powered air-purifying respirators (PAPRs) (Fig. 1). The tracheal window was made by the surgeon using closed suction with a viral filter. After exposure of the anterior tracheal wall, a No. 7.0 
Fig. 1. Patient positioning and preparation in the negative-pressure operating room.
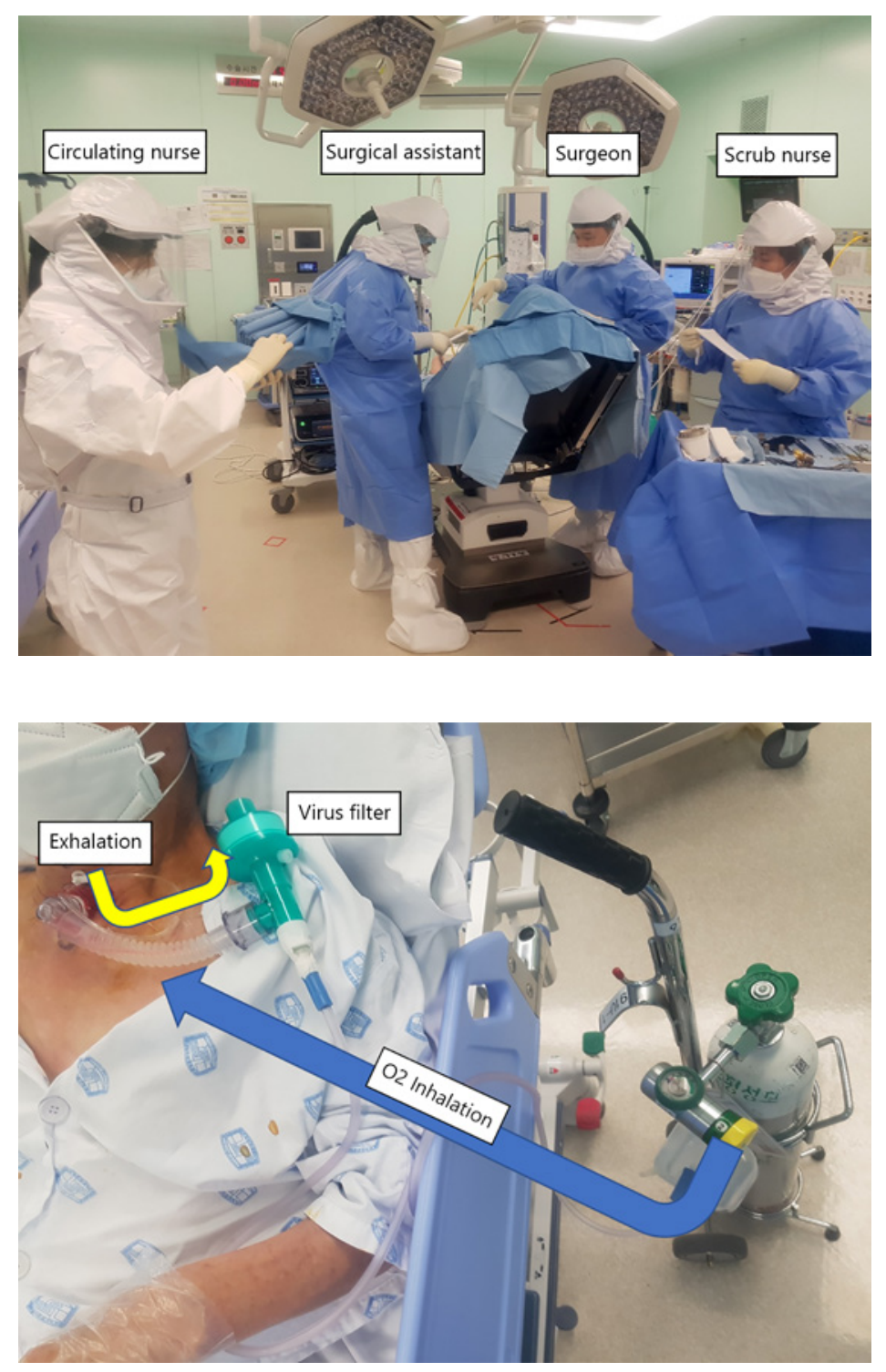

Fig. 2. Using a closed ventilation system with a virus filter to transfer the patient to the ICU negative-pressure room.

cuffed tracheotomy tube was carefully inserted. The tracheotomy tube cuff was inflated and closed-circuit ventilation was connected to the tracheotomy tube (Fig. 2). A week later, after the COVID-19 PCR results were reported as negative, total laryngectomy was performed.

\section{For the Physician}

Several reports on tracheotomy performed during the SARS, MERS, and COVID-19 eras are summarized in Table 2. During the COVID-19 pandemic, it may be safe to plan surgery considering the following: first, it needs to be determined whether airway obstruction is critical and if tracheotomy is required. Depending on the situation, tracheotomy can be defined as emergent (performed within 2-3 min), timely (5-10 min), or elective. Second, the risk of infection must be considered, especially as to whether the patient is known to be infected with COVID-19, at high risk of COVID-19, or has another infection. If COVID-19 infection cannot be ruled out, the highest level of PPE is required for tracheotomy [2]. A 


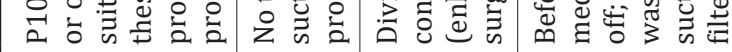

$\stackrel{\breve{.}}{\Xi}$<smiles>[C]1C[C@H]2CC[C@H]12</smiles>

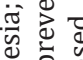

远

苟远

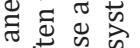
$\frac{\pi}{\pi} \stackrel{\oplus}{0} 心$ 范

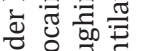

蓉:

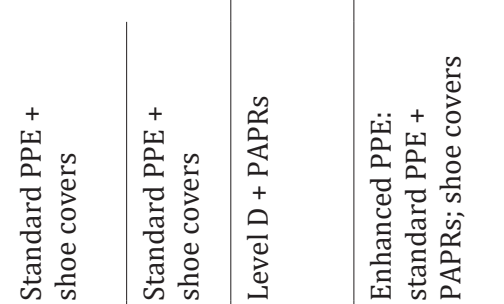

索

$\frac{0}{\bar{z}}$

高

हี

产

䓠

d.

苞

ปัँ

巳

$\Xi$

응

:

ह

है

过

峁

:

范

言

«

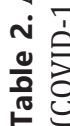
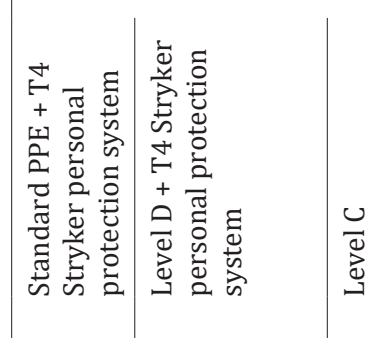

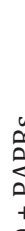

$\frac{2}{2}$
$\frac{1}{2}$
+
0
0
0
0
0

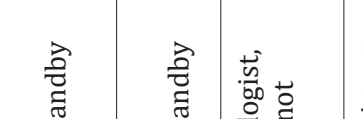

i

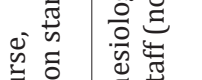

芯密

至

苯莙

至焉

已

元 论出

हुँ है

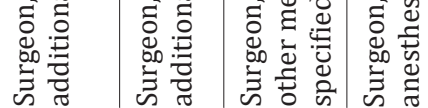

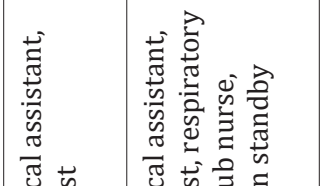

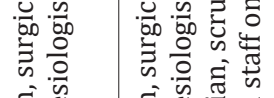

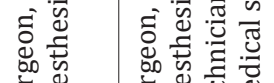

要

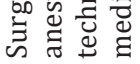

0
0
0
0
0
0
0
0
$z$

in

需

氙.

离芯芯

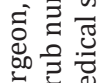

紊芯芯

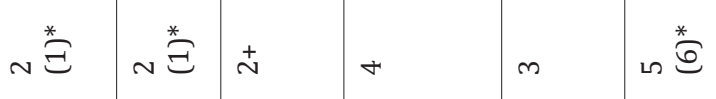

I

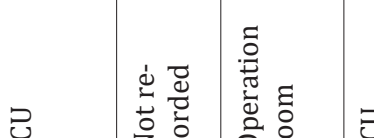

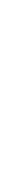

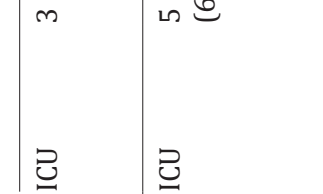

马

$+\stackrel{*}{\stackrel{*}{(}}$

ธี

范

\begin{tabular}{l|l}
0 & 0 \\
0 & 0
\end{tabular}

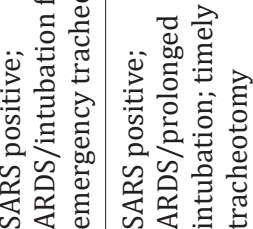

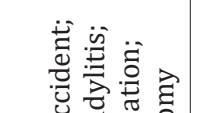

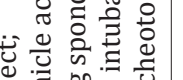

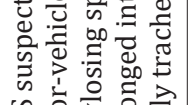

娄

분응 는

ลे تٓ

u

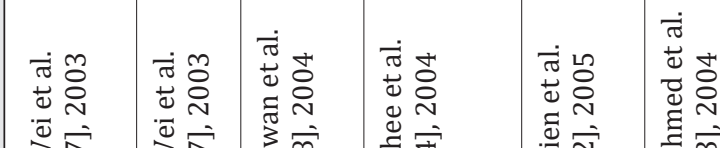

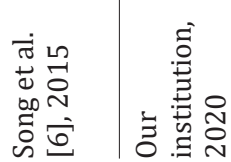

产完

胃 *

\%

敨

के

言, 嗃

우융

苋

宽

들

उ.

जे

$\overleftrightarrow{\square} \frac{0}{60}$

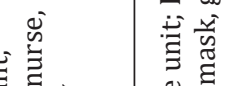

ซึ้ นุ

究

或

氙

है

ธิ

के के

$\tau$ ○

突

के वे

ळे

둥음

ขึ

安茞

苞吅离

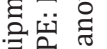

苛命

要

बे च

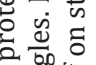

줄

क्षेّ हैं

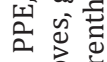
음 
screening test for COVID-19 prior to the procedure is mandatory in patients scheduled for elective tracheotomy [3].

PPE is the most effective preventative measure. The Centers for Disease Control and Prevention classify PPE from level A to level D; the latter includes coveralls, an N95 mask, goggles, shoe covers, two pairs of gloves, and an optional face shield. The principal difference between levels $C$ and $D$ is that level C uses PAPRs to protect the wearer from airborne substances, and the clothing is chemically resistant. The assigned protection factors are 10 for the N95 mask and $25 \sim 1,000$ for PAPRs; it is thus best to wear level D PPE + PAPRs or level C PPE to prevent airborne transmission of COVID-19 [1]. Difficulties related to the wearing of PPE that commonly arise during surgery include difficulties in communication due to the fan noise of the PAPR, awkward handling of surgical instruments, and the limited visual field. The patient flow plan to the operating room should also be checked. Patients should be transferred to the nearest negative-pressure facility (a ward, an intensive care unit, or an emergency room) via the shortest possible route, avoiding contact with unprotected medical staff and uninfected individuals [4].

Generally, tracheotomy does not require high-level surgical skills, but an experienced surgical team (anesthesiologist, surgeon, and surgical assistant) is necessary given the risks of the COVID-19 pandemic [5]. Because communication between surgical staff members wearing PAPRs is challenging during surgery, discussions should be conducted in advance to the extent possible. If an unexpected emergency situation arises, such as massive bleeding from the innominate artery during surgery, this cannot be appropriately managed if all necessary instruments have not been previously prepared in the negative-pressure operating room. Surgical experience and anatomical knowledge are critical [6] to keep the operating time as short as possible and thus minimize complications as well as aerosol exposure time [7]. To reduce the risk of airborne transmission, a closed suction system fitted with a viral filter and the use of minimal suction are recommended [8]. During the procedure, electrical cauterization should be kept to a minimum, cold instruments used whenever possible, and bleeding controlled with ties or gauze compression. In patients under general anesthesia, the anesthesiologist should stop mechanical ventilation temporarily before the trachea is opened, to prevent aerosols from spreading. A cuffed, non-fenestrated tracheotomy tube and a heat and moisture exchanger fitted with a viral filter should be used.

A previous study found that the COVID-19 virus can spread for as long as median 20.0 (16.0-23.0) days after COVID-19 onset. It is therefore advisable to replace or extubate the tube when the patient becomes negative for COVID-19 [9]. If the patient becomes positive for COVID-19, level D PPE is needed to protect medical staff during changing of the tracheotomy tube. As aerosols can spread during this maneuver, the tube should be changed only when necessary and only in a safe setting, such as a negative-pressure room. The tracheotomy tube should be removed and inserted gently, considering the curvature of the tube. As soon as the new tube is in place, the obturator should be replaced with an inner cannula and the balloon inflated with a $10-\mathrm{mL}$ syringe to prevent aerosol leakage. The closed ventilation system is then reconnected with a virus filter. The issues that need to be considered prior to tracheotomy in patients at high risk of COVID-19 are summarized below in the Key Points.

\section{Key Points}

1 CO (condition of patient): test for COVID-19 infection and assess the airway.

2 R (route): determine the safest route to the operating room.

30 (operation team): interprofessional team competencies are essential.

$4 \mathrm{~N}$ (negative pressure): a negative-pressure isolation facility will reduce infection risk.

5 A (appliance): PPE is required for all involved staff. 
Lee et al.: Tracheotomy during the COVID-19 Pandemic

\section{Conclusion}

A detailed protocol is needed to protect medical staff who perform tracheotomy under COVID-19 pandemic conditions; consideration of the patient's condition, the experience of medical staff members, and available facilities and equipment are required.

\section{Conflict of Interest Statement}

The authors declare no conflict of interest.

\section{Funding Sources}

This work was supported by grants from the Eunpyeong St. Mary's Hospital, College of Medicine, The Catholic University of Korea.

\section{Author Contributions}

Dong-Hyun Lee, Subin Kim, Ji-Sun Kim, Byung Guk Kim, Ki-Hong Chang, Jun-Ook Park: all those designated as authors should meet all four criteria for authorship, and all who meet the four criteria should be identified as authors. Substantial contributions to the conception or design of the work; or the acquisition, analysis, or interpretation of data for the work; AND drafting the work or revising it critically for important intellectual content; AND final approval of the version to be published; AND agreement to be accountable for all aspects of the work in ensuring that questions related to the accuracy or integrity of any part of the work are appropriately investigated and resolved.

\section{References}

1 Givi B, Schiff BA, Chinn SB, Clayburgh D, Iyer NG, Jalisi S, et al. Safety Recommendations for Evaluation and Surgery of the Head and Neck During the COVID-19 Pandemic. JAMA Otolaryngol Head Neck Surg. 2020 Mar; 146(6):579.

2 Tien HC, Chughtai T, Jogeklar A, Cooper AB, Brenneman F. Elective and emergency surgery in patients with severe acute respiratory syndrome (SARS). Can J Surg. 2005 Feb;48(1):71-4.

3 Ahmed N, Hare GM, Merkley J, Devlin R, Baker A. Open tracheostomy in a suspect severe acute respiratory syndrome (SARS) patient: brief technical communication. Can J Surg. 2005 Feb;48(1):68-71.

4 Chee VW, Khoo ML, Lee SF, Lai YC, Chin NM. Infection control measures for operative procedures in severe acute respiratory syndrome-related patients. Anesthesiology. 2004 Jun;100(6):1394-8.

5 Tay JK, Khoo ML, Loh WS. Surgical Considerations for Tracheostomy During the COVID-19 Pandemic: Lessons Learned From the Severe Acute Respiratory Syndrome Outbreak. JAMA Otolaryngol Head Neck Surg. 2020 Mar; 146(6):517.

6 Song JH, Kim HC, Kang JG, Roh DH. Tracheotomy in Middle East Respiratory Syndrome: report of a Case. J Clinical Otolaryngol. 2015;26(2):301-6.

7 Wei WI, Tuen HH, Ng RW, Lam LK. Safe tracheostomy for patients with severe acute respiratory syndrome. Laryngoscope. 2003 Oct;113(10):1777-9.

8 Kwan A, Fok WG, Law KI, Lam SH. Tracheostomy in a patient with severe acute respiratory syndrome. Br J Anaesth. 2004 Feb;92(2):280-2.

9 Zhou F, Yu T, Du R, Fan G, Liu Y, Liu Z, et al. Clinical course and risk factors for mortality of adult inpatients with COVID-19 in Wuhan, China: a retrospective cohort study. Lancet. 2020 Mar;395(10229):1054-62. 